\title{
An effective deep-inspiration breath-hold radiotherapy technique for left-breast cancer: impact of post-mastectomy treatment, nodal coverage, and dose schedule on organs at risk
}

This article was published in the following Dove Press journal:

Breast Cancer - Targets and Therapy

14 June 2017

Number of times this article has been viewed

\section{Lynsey Rice ${ }^{1,2}$}

Christy Goldsmith ${ }^{1,2}$

Melanie ML Green ${ }^{2}$

Susan Cleator ${ }^{1,2}$

Patricia M Price ${ }^{1,2}$

'Department of Radiation Oncology, The Harley Street Clinic, ${ }^{2}$ Department of Surgery and Cancer, Imperial College London, London, UK
Correspondence: Lynsey Rice Radiotherapy Research and Development Lead, Department of Radiation Oncology, The Harley Street Clinic, 8I Harley Street, London, WIG 8PP, UK

$\mathrm{Tel}+442079357700$ (Extension 68338)

Fax +442072999420

Email lynsey.rice@hcahealthcare.co.uk
Background: We developed, applied, and prospectively evaluated a novel deep-inspiration breath-hold (DIBH) screening and delivery technique to optimize cardiac sparing in left-breast radiotherapy (RT) at our clinic. The impact of set-up and dose variables upon organs at risk (OAR) dose in DIBH RT was investigated.

Methods and materials: All patients with left-breast cancer referred between 2011 and 2014 of all disease stages, set-up variations, and dose prescriptions - were included. Radiographers used simple screening criteria at CT simulation, to systematically assess patients for obvious DIBH benefit and capability. Selected patients received forward-planned intensity-modulated RT (IMRT) based on a DIBH CT scan. A 3D-surface monitoring system with visual feedback assured reproducible DIBH positioning during gated radiation delivery. Patient, target set-up, and OAR dose information were collected at treatment.

Results: Of 272 patients who were screened, 4 withdrew, 56 showed no obvious advantage, and 56 showed benefit but had suitability issues; 156 patients were selected and successfully completed DIBH treatment. The technique was compatible with complex set-up and optimal target coverage was maintained. Comparison of free-breathing (FB) and DIBH treatment plans in the first five patients enrolled confirmed DIBH reduced heart radiation by $\sim 80 \%$ $(p=0.032)$. Low OAR doses were achieved overall: the mean (95\% confidence interval $[\mathrm{CI}])$ heart dose was 1.17 (1.12-1.22) Gy, and the mean ipsilateral lung dose was 5.26 (5.01-5.52) Gy. Patients who underwent a standard radiation schedule (40 Gy/15\#) after breast-conserving surgery had the lowest OAR doses: post-mastectomy treatment, simultaneous supraclavicular (SCV) node coverage, and alternative dose schedule ( $50 \mathrm{~Gy} / 25 \#)$ were interrelated variables associated with increased OAR risk and compromised ipsilateral lung dose constraints.

Conclusion: The DIBH technique was successfully implemented and resulted in optimally low heart radiation. All patients who demonstrate sufficient DIBH technique at planning CT are now offered DIBH RT at our clinic. Patients with more advanced disease, particularly those with additional pulmonary risk factors, warrant additional focus to improve lung sparing.

Keywords: breast cancer, radiotherapy, deep-inspiration breath-hold, organs at risk, adverse effects

\section{Introduction}

With the improvement in prognosis for patients with breast cancer, reducing long-term toxicity from treatment has become increasingly important. Left-breast radiotherapy 
(RT) usually incurs incidental dose delivery to the heart and lungs, which are treated as organs at risk (OAR). Heart irradiation increases the risk of radiation-induced heart disease and major coronary events (MCE), with a relative increase in MCE risk of $7.4 \%$ per Gray of mean heart dose received. ${ }^{1}$ As no apparent threshold has been determined for radiation-induced $\mathrm{MCE}$, it implies that there is no "safe" limit; therefore, the dose to the heart should be kept as low as possible. Moreover, pulmonary toxicity from breast RT occurs. Documented clinical side effects include reduced lung function, radiological abnormalities, radiation pneumonitis, fibrosis, and lung cancer. ${ }^{2-5}$

In free-breathing (FB) tangential left-breast RT, the heart typically receives a mean dose (MD) of 1.7-9.0 Gy. ${ }^{6}$ Many studies have shown that deep-inspiration breath-hold (DIBH) can spare the heart during irradiation of the left breast, reducing heart MD by $26.2-75.0 \%$, to $\sim 0.7-5.0 \mathrm{~Gy}{ }^{6-16}$ The use of intensity-modulated RT (IMRT) may enhance reduction of heart dose. ${ }^{8,13}$ Reductions of lung dose have also been reported with DIBH. ${ }^{9,10,13-15,17,18}$ Further advantages of DIBH, achieved through the elimination of breathing motion - and, therefore, minimization of the need to compensate for ontreatment uncertainties - are improvements to RT accuracy and dose distribution. ${ }^{19}$ However, the feasibility and efficacy of DIBH technique may be challenged by individual patient anatomical differences, resulting in variable OAR sparing with DIBH. Lack of patient suitability for DIBH technique and DIBH positioning reproducibility may additionally hinder DIBH radiation delivery.

The determination of patient benefit and capability, as well as optimal positioning reproducibility and verification methods, are required to optimize DIBH. ${ }^{6} \mathrm{~A}$ further need is to discriminate patient-individualized RT variables - including factors such as type of surgery and inclusion of regional lymph nodes (and, therefore, corresponding RT-field size and shape), or radiotherapy dose regimen (e.g. standard vs hypofractionated regimens) - on OAR in DIBH RT. Studies carried out in FB RT have demonstrated that factors such as dose/fractionation schedule, simultaneous supraclavicular (SCV) coverage, and post-mastectomy (Post-M) chest-wall treatment increase OAR. ${ }^{3,20-22}$ In DIBH RT, the situation is less clear. For example, one study showed increased lung irradiation for patients requiring periclavicular field irradiation compared with those who did not; ${ }^{18}$ one study of patients requiring regional nodal irradiation showed that the lowest heart and lung doses were achieved with moderate DIBH $(\mathrm{mDIBH})$ and tangential field IMRT technique; ${ }^{13}$ two studies have indicated that heart sparing may be greater with DIBH when regional nodes are included; ${ }^{23}$ and one small study indicated that hypofractionated regimen resulted in lower OAR doses. ${ }^{17}$

In this article, we describe the first-phase implementation of a novel DIBH technique which was designed to incorporate DIBH prior best practice. ${ }^{6,7}$ The technique comprised of a simple pretreatment screening protocol that enabled radiographers to make a quick, pragmatic, and systematic assessment of patients with left-breast cancer for obvious DIBH benefit and suitability, combined with qualitative individualized tangential IMRT planning with low OAR constraints, and a 3D-surface-imaging gated RT delivery protocol for DIBH positioning reproducibility assurance. The feasibility and efficacy of the technique were prospectively assessed through clinical application and measurement of OAR dose metrics at planning. The impact of RT variables on OAR risk was subsequently investigated by comparison of OAR doses in patient subgroups defined by dose schedule and RT set-up variations.

\section{Methods and materials}

\section{Study and ethics}

A novel technique incorporating DIBH for left-breast RT was implemented as a quality-improvement initiative in the Harley Street Clinic, where it is mandated policy to audit clinical outcomes under the governance of HCA Healthcare UK. All patients gave written informed consent for treatment and subsequent analysis, and publication of the data contained in this study was approved by HCA Healthcare UK, which has confirmed the study was undertaken in accordance with its quality, safety, and clinical governance frameworks throughout.

All patients with left-sided breast cancer due to undergo adjuvant RT between September 2011 and December 2014 were initially included and systematically screened for suitability and benefit of the DIBH technique. Feasibility and efficacy of the novel protocol were prospectively assessed, where the study cohort was defined as all consecutive patients who underwent left-breast DIBH RT treatment in the study period. RT variables and OAR data were collected at the time of treatment planning, and patients were assigned a study number for subsequent anonymized analysis.

\section{Simulation and patient selection}

CT simulation scans were performed by radiographers using a GE Lightspeed scanner RT 16 (General Electric; Fairfield, CT, USA), with the patient in the supine position on a Quest RT4543 breast-board (Q-Fix systems; Avondale, PA, USA). The breast-board was angled from $5^{\circ}$ to $25^{\circ}$, with the aim of leveling each individual patients' chest as horizontal as 
possible - for the vast majority of patients, a $15^{\circ}$ angle was used. Both arms were elevated and supported, unless simultaneous SCV node irradiation was required, wherein only the left arm was raised and supported. A customized, pliable, $1 \mathrm{~cm}$ thick bolus material (St Bartholomew's Radiotherapy Department, London, UK) was applied to chest-wall/scar for Post-M patients when considered necessary. Standard patient positioning was carried out using skin markers, room laser alignment, and breast-board scale positioning before acquisition of the initial free-breathing (FB) CT scan. Scans were acquired with a $2.5 \mathrm{~mm}$ slice width from $2.0 \mathrm{~cm}$ above the sternal notch (or covering the shoulder for SCV node involvement) to $4.0 \mathrm{~cm}$ below breast tissue.

At the time of simulation, radiographers determined patient benefit and capability for DIBH technique using previously described criteria, ${ }^{24}$ as outlined in Table 1 . The screening method was designed to be a quick and pragmatic way of determining patients who demonstrated obvious heart risk (heart within FB RT treatment field), easily performed good DIBH technique, and showed clear benefit of the DIBH technique (heart moves away from RT treatment field in DIBH). In the assessment sequence, cardiac position was initially assessed on FB CT simulation scan. The scanner software was used to place a line across the back edge of the clinical field placement on the central axis CT slice. Patients were considered "at risk" if cardiac tissue crossed into the target area on any point slice

Table I Screening criteria used for DIBH technique

\begin{tabular}{|c|c|}
\hline ecklist & tt \\
\hline $\mathrm{DIBH}$ & $\begin{array}{l}\text { - Heart tissue considered " } \mathrm{a} \\
\text { planning CT treatment fiel } \\
\text { be anatomically displaced d }\end{array}$ \\
\hline $\begin{array}{l}\text { No alternative available } \\
\text { method to improve } \\
\text { planning dosimetry }\end{array}$ & $\begin{array}{l}\text { - Other techniques e.g. multi-leaf } \\
\text { collimation (MLC) considered unsuitable/ } \\
\text { inferior }\end{array}$ \\
\hline $\begin{array}{l}\text { Adequate DIBH chest } \\
\text { breathing reproducibility }\end{array}$ & $\begin{array}{l}\text { - Patient instructed in } 20 \text { second DIBH } \\
\text { and supervised through several practices } \\
\text { - Patient able to follow breath-hold } \\
\text { instructions } \\
\text { - Regular rib cage rise and fall seen and } \\
\text { minimal displacement of anterior tattoo } \\
\text { on breast board scale }\end{array}$ \\
\hline $\begin{array}{l}\text { No patient specific } \\
\text { factors that would } \\
\text { compromise DIBH set-up } \\
\text { reproducibility }\end{array}$ & $\begin{array}{l}\text { - Sufficient shoulder movement, no arm } \\
\text { adjustment required to pass through CT } \\
\text { scanner, comfortable lying flat, stable } \\
\text { breast tissue without need for additional } \\
\text { support etc. }\end{array}$ \\
\hline $\begin{array}{l}\text { Benefit of D } \\
\text { confirmed }\end{array}$ & $\begin{array}{l}\text { - Planning CT in DIBH shows heart } \\
\text { displacement away from treatment field }\end{array}$ \\
\hline
\end{tabular}

Notes: Adapted from Rice L, Harris S, Green MML, Price PM. Deep inspiration breath-hold (DIBH) technique applied in right breast radiotherapy to minimize liver radiation. BJR Case Reports. 2015;2:20150038. ${ }^{24}$

Abbreviation: DIBH, deep-inspiration breath-hold.
(Figure 1A). These patients were then instructed in voluntary DIBH technique, and supervised while they practiced it several times. Patients were asked to relax and breathe out, then breathe in to a higher level than normal but within comfortable limits, and to hold their breath for 20 seconds. Chest breathing reproducibility was visually assessed and anterior tattoo displacement was measured. If the radiographers considered that the patient demonstrated good DIBH technique, a second CT simulation scan was acquired during DIBH, and cardiac position was re-assessed as per FB CT (Figure 1B). An appointment time of 60 minutes was allotted for simulation to allow for breath-hold coaching and practice, assessment, and an extra scan in DIBH if required. The opinion of a clinical oncologist was sought if there was any doubt regarding patient benefit or suitability. Selected patients were given a written patient information sheet explaining the DIBH technique expected during their treatment course.

\section{DIBH treatment planning}

CT data sets were transferred to the Eclipse ${ }^{\mathrm{TM}}$ planning system (Varian Medical Systems Palo Alto, CA, USA), and forward-planned IMRT plans were generated by a dosimetrist in conjunction with the treating clinician. For the first five selected patients, plans were also prepared on FB simulation scans using the same planning parameters, for subsequent comparison of cardiac and lung dosimetry.

For patients with intact breast tissue following breastconserving surgery (BCS), the clinical target volume (CTV) was defined as the soft tissues of the whole breast down to the deep fascia, but not including muscle and rib cage or overlying skin/excision scar. For Post-M patients ( \pm implant reconstruction), the institutional practice followed was to create a CTV which encompassed the skin flaps from $5 \mathrm{~mm}$ below the skin surface and included the soft tissues down to the deep fascia, but did not include underlying muscle and the rib cage, utilizing anatomical landmarks for chest-wall localization. Planning target volumes (PTV) were generated by uniformly expanding the CTV with a $1 \mathrm{~cm}$ margin. For patients who required simultaneous SCV nodal irradiation (+NODES), the inferior SCV field border was matched to the upper tangential field border and the medial border was placed at the edge of the clavicle and along the outer edge of the cervical vertebrae. The superior border was defined by the $\mathrm{C5} / \mathrm{C} 6$ interspace, and the lateral border was positioned to cover the most superior/medial clip of the axillary nodal dissection, or two-thirds along the clavicle. The border was extended laterally to cover the humeral head if the axilla required treatment. The internal mammary chain nodes were not included in treatment fields. 

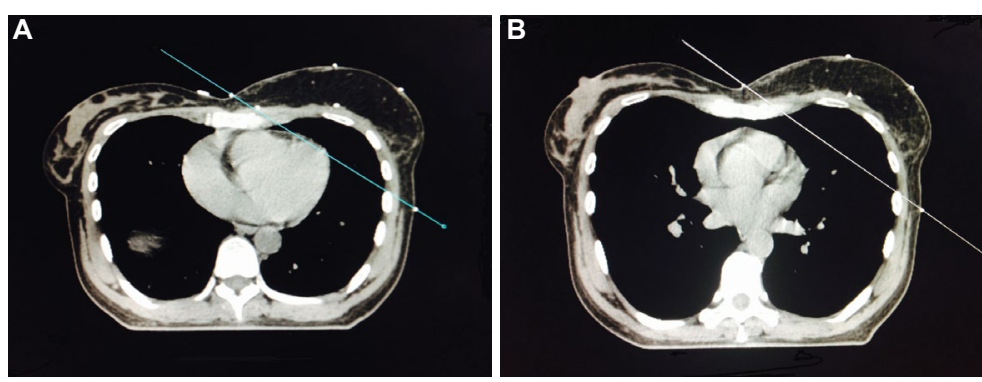

Figure I Central axis slice of CT planning scans acquired in free-breathing $(\mathbf{A})$ and deep-inspiration breath-hold (B) in a representative patient showing heart position in relation to the back edge of the field placements. The diagonal line represents the back edge of the treatment area.

The whole heart was outlined to the extent of the pericardial sac, as per the SCOPE1 trial methods. ${ }^{25}$ The superior point was defined where the pulmonary trunk and the right pulmonary artery were seen as separate structures. The major blood vessels (superior to the organ) and the inferior vena cava (toward the inferior extent of the heart) were excluded and the left anterior descending (LAD) artery was included in the heart contour as a whole. Lungs were automatically contoured using the planning system. Plans were primarily evaluated using the mandatory cardiac constraint of no more than $10 \%$ of heart volume to receive $13 \mathrm{~Gy}$ or more (V13Gy $<10 \%)$ and were optimized to deliver $95 \%-105 \%$ of the prescribed dose to the PTV. Optimal dose constraints were: heart V13Gy $<2 \%$, mean dose (MD) $<3 \mathrm{~Gy}$; ipsilateral lung (ilung) V18Gy $<10 \%$, $\mathrm{MD}<6 \mathrm{~Gy}$; and contralateral lung (clung) $\mathrm{V} 2.5 \mathrm{~Gy}<3 \%$, $\mathrm{MD}<1 \mathrm{~Gy}$. Doses prescribed were at the treating clinicians' discretion: either the standard UK dose schedule of $40.05 \mathrm{~Gy}$ in 15 fractions $(40 \mathrm{~Gy} / 15 \#)$, or the alternative dose schedule of $50 \mathrm{~Gy}$ in 25 fractions $(50 \mathrm{~Gy} / 25 \#)$, was applied. A fieldin-field breast tangential approach was used by integrating a maximum of 6 or 10 megavoltage (MV) beams, limiting the latter for skin sparing. Patients requiring SCV node treatment were planned using an anterior field to the maximum dose (Dmax). Beam-on times were limited or split to incorporate the 20 second breath-hold DIBH technique.

\section{D surface imaging during treatment}

RT was delivered using a Varian Trilogy ${ }^{\mathrm{TM}}$ Linac. During RT, a 3D surface imaging system (AlignRT ${ }^{\circledR}$ Beam-Hold; VisionRT Ltd, London, UK) was used to achieve a stable, reproducible breath-hold position, track real-time patient motion in six degrees-of-freedom, and automatically gate radiation delivery. Video goggles/tablet visualization were used to provide the patient with visual feedback and coaching (Figure 2) for DIBH reproducibility and stability. ${ }^{26,27} \mathrm{~A}$ 30 to 45 minute in-room appointment time (which included patient dressing and undressing, set-up, daily imaging, DIBH practice, and subsequent gated RT delivery) was allotted for treatment Day 1 to allow a DIBH/AlignRT practice period prior to radiation delivery. For subsequent treatment days, a 20 to 30 minute in-room appointment time was allocated, which is double the 10 to 15 minute time allocated for conventional FB radiation delivery.

\section{Treatment verification}

For patients who did not require SCV node irradiation, daily pretreatment medial field verification was carried out using Varian PortalVision ${ }^{\mathrm{TM}}$, with extended posterior collimator jaw (maximum $2 \mathrm{~cm}$ ) to visualize heart position on electronic portal images (EPI). On occasions when field placement was out-of-tolerance, the AlignRT real-time delta (RTD) values were used with EPI to correct patient positioning. For patients with extended SCV target field, daily kilovoltage $(\mathrm{kV})$ images were acquired with the Varian On-Board Imager ${ }^{\circledR}$. All pretreatment images were matched online to verify field positioning before beam delivery, using a tolerance of $0.5 \mathrm{~cm}$.

\section{Data analysis}

Heart and lung dose metrics were generated by the Eclipse planning system. Statistical analysis was performed using GraphPad Prism ${ }^{\circledR}$ version 6, applying relevant two-tailed tests, and using a significance level of $p<0.05$.

\section{Results}

\section{Patients}

A summary of study patients is given in Table 2. Of 272 patients referred for left-breast adjuvant RT, four patients subsequently withdrew from RT, and 112 patients were excluded from DIBH by suitability screening. For 56 patients, exclusion was either because the heart did not cross into the target field in FB, or because the anatomical changes observed in DIBH did not result in the heart moving out of the target field (patient showed no obvious benefit). A further 56 patients were excluded due to DIBH technique feasibility issues, giving a feasibility rate of 156/272 (79\%). Reasons DIBH was considered unfeasible included insufficient ability to demonstrate good breath-hold technique (28 patients), 
A

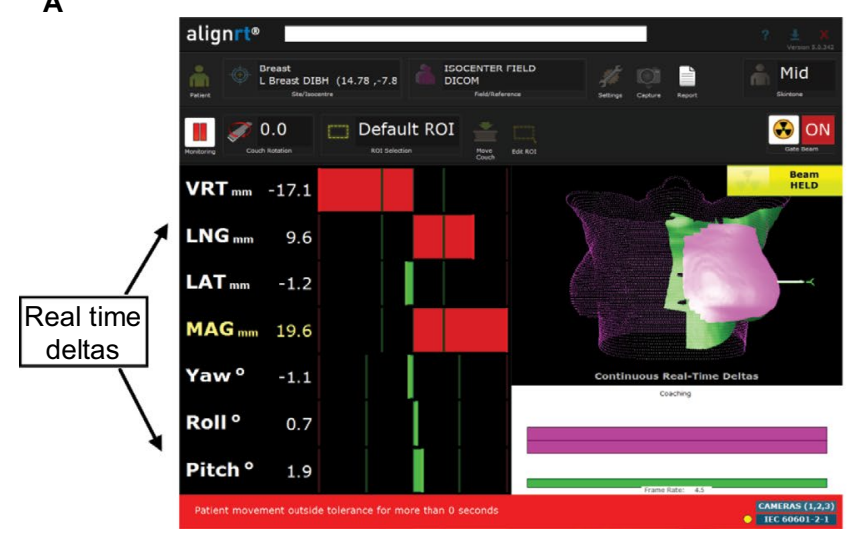

B

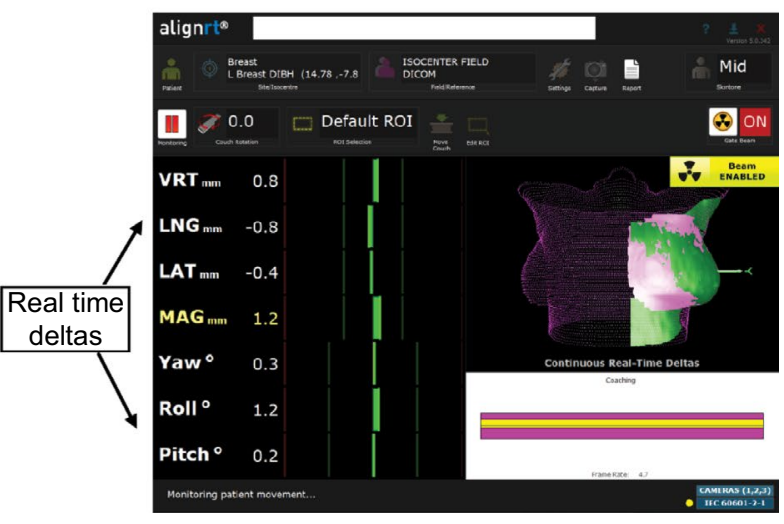

Figure 2 Screen shots of the AlignRT ${ }^{\circledR}$ workstation showing real-time delta (RTD) values (left-hand side) and the patient coaching bar (bottom right) when patient positioning is out-of-tolerance to DIBH positioning and the radiation beam is held (A); and when patient positioning is within set tolerance and the beam is enabled (B).

Table 2 Study patients

\begin{tabular}{|c|c|c|}
\hline \multicolumn{2}{|c|}{ Patient diagnosis and treatment details } & $\begin{array}{l}\text { Number of } \\
\text { patients }\end{array}$ \\
\hline \multicolumn{3}{|c|}{ - Screened N = 272 (100\%) } \\
\hline \multicolumn{3}{|c|}{ - Excluded $N=116(43 \%)$} \\
\hline & No obvious benefit & $56(21 \%)$ \\
\hline & Technique feasibility issues & $56(21 \%)$ \\
\hline & Patient withdrawal & $4(1.5 \%)$ \\
\hline \multicolumn{3}{|c|}{ - Selected $N=156(57 \%)$} \\
\hline \multirow[t]{2}{*}{ Tumor size } & Median 22 mm (range 4-80 mm) & 152 \\
\hline & Unknown/Entire breast & 4 \\
\hline \multirow[t]{6}{*}{ T stage } & TIS & 10 \\
\hline & $\mathrm{TI}$ & 55 \\
\hline & $\mathrm{T} 2$ & 73 \\
\hline & T3 & 15 \\
\hline & $\mathrm{T} 4$ & 2 \\
\hline & TX & 1 \\
\hline \multirow[t]{2}{*}{$\mathrm{N}$ stage } & No & 114 \\
\hline & $\mathrm{N}+$ & 42 \\
\hline \multirow[t]{2}{*}{ M stage } & Mo & 152 \\
\hline & MI & 4 \\
\hline \multirow[t]{2}{*}{ Prior surgery } & Breast conserving surgery (BCS) & 116 \\
\hline & Mastectomy \pm reconstruction & 40 \\
\hline \multirow[t]{2}{*}{ SCV nodes } & Not required & 137 \\
\hline & + NODES & 19 \\
\hline \multirow[t]{2}{*}{ Schedule } & 40 Gy in 15 fractions $(40 \mathrm{~Gy} / 15 \#)$ & 143 \\
\hline & $50 \mathrm{~Gy}$ in 25 fractions ( $50 \mathrm{~Gy} / 25 \#)$ & 13 \\
\hline
\end{tabular}

Abbreviation: SCV, supraclavicular.

poor understanding (13 patients), anxiety (12 patients), large mobile breast tissue requiring additional immobilization (2 patients), and refusal to have tattoos (1 patient).

Of the 156 selected patients, 104 were post-BCS patients who were prescribed $40 \mathrm{~Gy} / 15 \#$ to the intact breast region (BCS+40Gy/15\#). For the remaining 52 patients, one or more of the following interrelated variables applied: patient was Post-M with/without implant reconstruction and/or bolus necessary for skin dose modulation; extended field was required to cover SCV nodes (+NODES); and/or patient was prescribed the alternative dose $50 \mathrm{~Gy} / 25 \#$ regimen. As expected, more patients who required SCV node irradiation were Post-M (consistent with more advanced disease); and more patients who required SCV coverage were prescribed the $50 \mathrm{~Gy} / 25 \#$ dose regimen (both chi-squared $p<0.0001$ ).

\section{Planning study}

Example digital reconstructed radiographs (DRR) generated from FB and DIBH planning CT scans are shown in Figure 3. Comparative dosimetry from plans generated on FB and DIBH scans for the first five selected patients are shown in Table 3. DIBH reduced heart V13Gy by $\sim 80 \%$, compared with FB (Wilcoxon $p=0.032$ ).
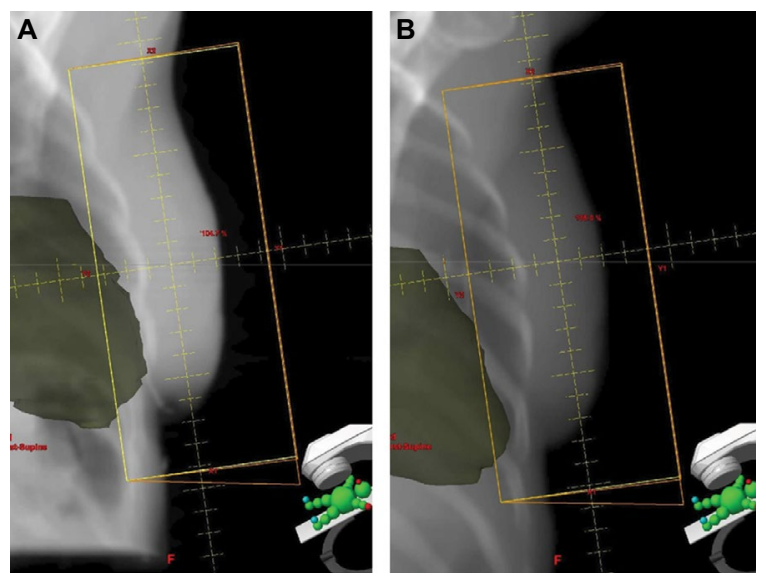

Figure 3 Digital reconstructed radiographs (DRR) generated from (A) freebreathing and (B) DIBH planning CT scans acquired from patient I. The heart (shaded) moves inferiorly and posteriorly away from the tangential field with DIBH. Abbreviation: DIBH, deep-inspiration breath-hold. 
Table 3 Comparative heart and ipsilateral lung (iLung) doses from FB and DIBH plans prepared for the first five selected patients, who were optimally planned on both FB CT scan and DIBH CT scan using the same planning protocol

\begin{tabular}{|c|c|c|c|c|c|}
\hline \multirow[t]{2}{*}{ Patients* } & \multirow[t]{2}{*}{ RT variables } & \multicolumn{2}{|l|}{ Heart VI3Gy } & \multicolumn{2}{|l|}{ iLung V20Gy } \\
\hline & & FB & DIBH & FB & DIBH \\
\hline 1 & BCS, 40 Gy/I5\# & 6.64 & 1.07 & 6.64 & 9.46 \\
\hline 2 & BCS, 40 Gy/I5\# & 0.7 & 0 & 10.84 & 8.4 \\
\hline 3 & BCS, 40 Gy/I5\# & 1.39 & 0.21 & 4.83 & 7.09 \\
\hline 4 & BCS, 40 Gy/I5\# & 0.73 & 0.24 & 4.6 & 6.8 \\
\hline 5 & Post-M, 50 Gy/25\# & 3.33 & 0.46 & $12.2 \mid$ & 10.7 \\
\hline Median $(95 \% \mathrm{Cl})$ & & $1.39(-0.57$ to 5.68$)$ & $0.24(-0.11$ to 0.9$)$ & 6.64 (3.47 to 12.17$)$ & 8.4 (6.46 to 10.52$)$ \\
\hline Wilcoxon & & & $p=0.032 *$ & & $p=0.63$ \\
\hline
\end{tabular}

Notes: *None of the first five patients had any implant, required bolus, or required SCV node irradiation. The first four patients were post-BCS patients who were prescribed $40 \mathrm{~Gy} / \mathrm{I5 \#}$. The fifth patient was Post-M and was prescribed $50 \mathrm{~Gy} / 25 \#$. OAR dose variations are attributed to individual differences in tumor size, position, and patient anatomy that affected field positioning.

Abbreviations: $\mathrm{BCS}$, breast-conserving surgery; $\mathrm{Cl}$, confidence interval; $\mathrm{DIBH}$, deep-inspiration breath-hold; $\mathrm{FB}$, free-breathing; OAR, organs at risk; Post-M, postmastectomy; RT, radiotherapy; SCV, supraclavicular.

\section{Treatment feasibility}

The DIBH screening protocol was quick and straightforward for radiographers to undertake. Reassuringly, the DIBH technique was found to be fully compatible with all planning and set-up requirements, including low OAR dose constraints, SCV nodal coverage, surgery status (BCS or Post-M, with or without reconstruction implant), and bolus application. All 156 selected patients complied well with the AlignRT-mediated DIBH RT technique and completed their prescribed RT course, giving a compliance rate of $100 \%$ for selected patients. An additional benefit was that all in-room appointment times reduced from 30 to 45 minutes on Day 1 to 10 to 20 minutes on subsequent treatment days.

\section{Dose to heart and lungs}

The mean $(95 \%$ confidence interval [CI]) heart MD for selected patients $(n=156)$ was $1.17(1.12-1.22)$ Gy and was below the optimal constraint of $3 \mathrm{~Gy}$ in all patients. The vast majority of patients (99.6\%) had a heart MD below 2 Gy: only 1 patient had a heart MD above $2 \mathrm{~Gy}$. The mean heart V13Gy was $0.17 \%(0.11 \%-0.24 \%)$, with $154 / 156$ (99\%) of patients below the optimal constraint of $2 \%$. The mean ilung MD was 5.26 (5.01-5.52) Gy. The optimal ilung MD constraint of 6 Gy was met in 116/156 (74\%) of patients and breached in 40/156 (26\%) patients. The ilung V20 was higher than optimal in 58/156 (37\%) of patients, where it ranged from $10 \%$ to $27 \%$. The mean clung MD was low at 0.16 $(0.14-0.19)$ Gy, with only $2 / 156(1 \%)$ of patients breaching the optimal constraint of $1.0 \mathrm{~Gy}$.

Heart and lung doses were examined in relation to dose and field set-up variables. Patients who were Post-M and/ or required concurrent SCV node coverage (Post-M and/ or +NODES) had significantly greater heart and ilung MD compared with BCS patients (regression analysis $p<0.0001$ ). Patients who received the $50 \mathrm{~Gy} / 25 \#$ regimen had significantly greater heart and ilung MD compared with patients who received the $40 \mathrm{~Gy} / 15 \#$ regimen (regression analysis $p<$ 0.0001 ). This was supported by Mann-Whitney $U$ comparisons for mean heart MD (1.52 Gy vs $1.14 \mathrm{~Gy}$, respectively $p<$ 0.001 ), and mean ilung MD (7.38 Gy vs 5.07 Gy respectively, $p=0.003)$. When patient subgroups were further examined (Figure 4), it was observed that patients who were Post-M and/or +NODES and/or received the $50 \mathrm{~Gy} / 25 \#$ schedule also typically breached optimal ilung dose constraints. Notably, all patients who were Post-M+NODES breached ilung MD constraints and had significantly higher ilung MD compared with BCS patients for both dose regimens (Kruskall-Wallis with Dunn's post hoc for multiple comparisons $p<0.05$; Figure 4B). Conversely, of the 40/156 (26\%) patients whose ilung MD was $>6 \mathrm{~Gy}$, uneven distribution for nodal coverage, Post-M treatment, and alternative dose schedule was observed: 14/19 (74\%) required SCV nodal coverage (+NODES), 20/40 (50\%) were Post-M, and 8/13 (62\%) received the $50 \mathrm{~Gy} / 25 \#$ dose schedule (all chi-squared $p<0.05$ ). Furthermore, for patients who were Post-M and/or +NODES, the increase in ilung MD was greater than might be expected when the $50 \mathrm{~Gy} / 25 \#$ dose schedule was compared with those who received the $40 \mathrm{~Gy} / 15 \#$ dose schedule: the ilung MD was 1.36-fold greater for BCS+NODES patients ( 7.73 vs 5.69 Gy); the ilung MD was 1.67-fold higher for Post-M patients (8.01 vs 4.82), and the ilung MD was 1.49 -fold higher for Post-M+NODES patients (10.33 vs 6.94 Gy).

\section{Discussion}

We developed and successfully applied a novel DIBH protocol incorporating DIBH/3D surface imaging/tangential IMRT in one of the largest patient series examined to date. To our knowledge, it is the first clinical study to 1) systematically implement a pre-screening protocol for all patients requiring left-breast RT to select patients for DIBH benefit and capability; 
A

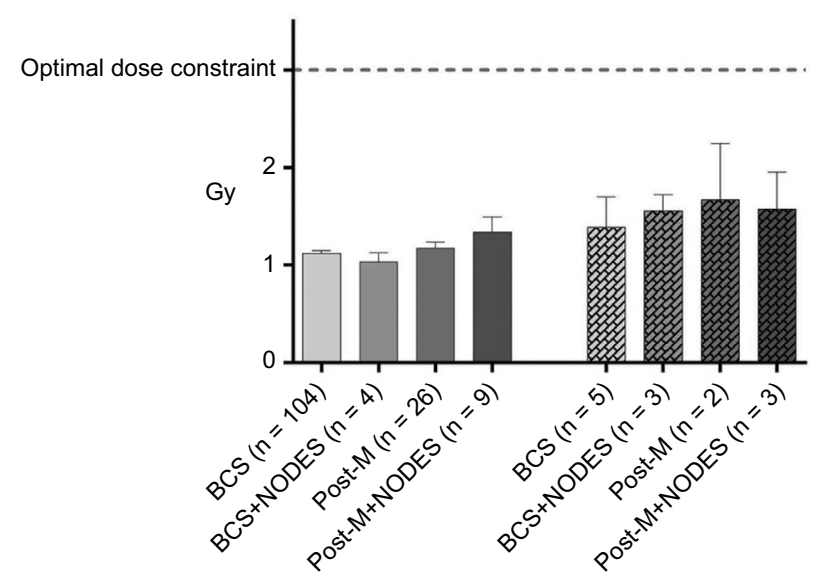

40 Gy/15\#
Heart mean dose

$50 \mathrm{~Gy} / 25 \#$
B

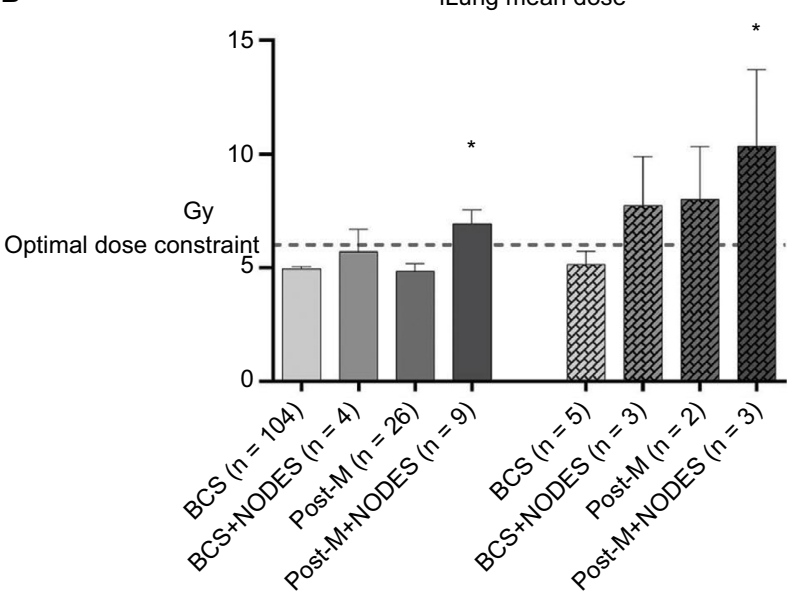

40 Gy/15\#

$50 \mathrm{~Gy} / 25 \#$

Figure 4 OAR doses of DIBH-treated patients. Bar charts showing (A) heart MD and (B) ilung MD of patients treated with DIBH RT $(n=156)$, with patients divided into subgroups by dose regimen $40 \mathrm{~Gy} / \mathrm{I5} \#$ (plain bars) versus $50 \mathrm{~Gy} / 25 \#$ (patterned bars) and field-set up variations BCS (light grey), BCS+NODES (mid grey), Post-M (dark grey) and Post-M+NODES (darkest grey). *Patient subgroups significantly different to BCS+40Gy/15\# patients.

Abbreviations: BCS, breast-conserving surgery; DIBH, deep-inspiration breath-hold; OAR, organs at risk; Post-M, post-mastectomy; SCV, supraclavicular.

and 2) to comprehensively evaluate the impact of dose schedule AND RT variables upon OAR doses. The main positive findings were: the pragmatic feasibility of the screening method; that all selected patients completed treatment with excellent compliance; and that the technique was compatible with low heart dose constraints and complex set-up, including SCV node coverage, without compromising qualitative target coverage. Moreover, patients received OAR doses lower than other reported DIBH methods. ${ }^{6,9-11,13,14,18,28,29}$ Nevertheless, the main clinical challenges of the technique included: incorporating patient selection into routine practice; the need for an additional planning CT scan at simulation for those "at risk" of cardiac toxicity; the financial and space implications for extra equipment required; the additional planning requirements to incorporate low OAR constraints and 20 second beam-on times; and the additional clinic time required for staff training, and longer appointment times to incorporate patient selection, patient coaching, DIBH radiation delivery, and daily quality assurance.

The main advantages of the selection protocol were that it was quick and inexpensive, requiring simple radiographer assessment at simulation to exclude patients who were considered unsuitable. In comparison with other screening methods, the key benefits were: only one simulation appointment was necessary; consultation with a radiation oncologist for assessment of patient benefit or suitability for DIBH treatment was not necessary; an additional CT scan was only carried out for those capable of the technique AND considered "at cardiac risk" - so that additional radiation dose for an additional CT scan was only incurred for suitable patients; and there was no need for full FB and DIBH treatment plan preparation and comparison in all patients. ${ }^{9,10,18,30,31}$ The main drawbacks of the screening protocol were: that it was only partially validated (whereby the first five selected patients were shown to have significantly reduced cardiac dose with DIBH compared with FB); it was not designed as a comprehensive or foolproof protocol to "correctly" select patients for optimal treatment; and the proportion of patients who were excluded on crude assessment of capability and risk/benefit was considered unnecessarily high. Although the $21 \%$ of patients excluded due to lack of benefit is similar to a recent report which used cardiac contact distance on DIBH planning CT for patient selection, ${ }^{31}$ the judgment of heart irradiation by infringement into the treatment field is acknowledged not to be a comprehensive way of assessing heart irradiation. This early-stage judgment in the initial stages of technique introduction was designed to assess maximum patient benefit. An additional $21 \%$ of patients who may have benefited were excluded due to DIBH feasibility issues. These weaknesses became a recognized area of targeted improvement ${ }^{6}$ as they indicate that, for some patients, potential beneficial reductions to heart dose - and, therefore, relative risk of MCE - may not have been recognized and minimized, and for other patients, MCE risk may be approximately doubled ${ }^{1}$ for reasons that are potentially surmountable. As a result of the experience gained in this study, and from recent data in the literature, our current practice has changed. All patients with left-breast cancer are offered DIBH treatment, and all patients are first scanned in FB. Those who are capable of "sufficient DIBH technique" then undergo repeat scanning in DIBH and undergo DIBH treatment. This process permits full validation and enables inclusion of patients whose DIBH benefits for cardiac sparing may be less obvious. Further, the new process has increased 
our suitability rates to $\sim 88 \%$. This is because patients are now not required to demonstrate "ideal" DIBH technique, since the experience gained in this study led to confidence that initial "sufficient" DIBH technique combined with AlignRT coaching on treatment Day 1 ensures patients become good at reproducible DIBH technique. However, patient feasibility issues such as anxiety, insufficient understanding of instructions, or requirements for additional immobilization aids (e.g. breast shells) do still mean that some patients are not suitable for DIBH.

Dual planning carried out in the first five selected patients provided some validation of the selection process, and gave confidence to continue selecting patients for DIBH treatment. Consistent with published evidence, heart dose was significantly reduced with DIBH in the first five patients - which was the primary aim of the DIBH technique. Although substantial inter-patient variability was seen in lung dose metrics with DIBH, where ilungV20Gy was reduced in two patients and increased in three patients, the mean ilungV20Gy for all five patients was reassuringly low as compared with other DIBH studies. ${ }^{6,7,10,14,28,32}$ The AlignRT system was a useful aid to set-up when used in conjunction with PortalVision and ensured accurate and reproducible positioning during DIBH. The advantages of the system are that it does not require extensive immobilization, incur additional radiation dose, or require the radiographer to control beam delivery and provides electronic real-time visual guidance to patients. Further benefits were that workload and patient training were also less cumbersome, treatment times remained relatively short, and patient comfort and compliance was good compared with other surrogates of breath-hold positioning. ${ }^{6,9,10,14,18,26,33,34}$

OAR doses obtained for the study patients $(n=156)$ were optimally low as compared with other DIBH studies. ${ }^{6,9-11,13,14,18,27,28}$ Heart doses were among the lowest reported for DIBH IMRT. ${ }^{6,8,13}$ Lung doses (ilung MD and ilung V20Gy) were also lower overall as compared with many other studies, ${ }^{9-14,18,28,29,32}$ although substantial inter-patient variability was observed. This is likely because of individual patient anatomy and target-field morphological variations, such as chest shape, lung size, breast shape, size, position, and mobility, which influences lung volume proportion within the tangential field and proximity to high-dose regions with inflation/deflation. Target-field (CTV and PTV) delineation, planning, and delivery methods also appear to have substantial influence. ${ }^{8-14,18,29}$ Importantly, our data confirm previous limited data that show dose schedule can contribute to increased OAR doses in DIBH treatment. ${ }^{17}$ This accords with data previously obtained in $\mathrm{FB}^{20,21}$ The $40 \mathrm{~Gy} / 15 \#$ schedule used in the majority of patients is standardly used in the UK, for which equivalent cosmetic outcome, reduced normal tissue effects, and reduced heart dose have been shown in comparison with the $50 \mathrm{~Gy} / 25 \#$ schedule in FB RT. ${ }^{20,35}$ The 50 Gy/25\# schedule is also considered acceptable and was used at the treating clinicians' discretion. Although our investigations of the impact of RT variables were limited by confounding interrelated factors and small patient subsets, our results show higher OAR risk for Post-M patients and/or those whose target field included SCV nodes (Post-M and/or +NODES patients) - a finding which may be considered unsurprising and intuitive with expanded radiation field. The most notable effect appeared to be on ilung dose, as ilung constraints were breached in all patients who were Post-M+NODES. Nevertheless, these patients were also more likely to receive the $50 \mathrm{~Gy} / 25 \#$ regimen, where the combination of Post-M+NODES treatment with the 50 Gy/25\# schedule appeared to have a synergistic effect on ilung dose, increasing it disproportionately compared with those that received the $40 \mathrm{~Gy} / 15 \#$ schedule. We postulate that this exacerbation is because of an overall increased RT dose, in combination with an increased volume of lung tissue with DIBH in the expanded target field when SCV nodes are simultaneously covered, and/or proximity of lung tissue to the high-dose region for Post-M patients. It may also be influenced by the RT beam arrangements and level of inspiration used for breath-hold. ${ }^{13}$ It is additionally possible that the method used for CTV/PTV definition may affect the degree of lung irradiation, and it is noteworthy that our institutional methods for Post-M CTV definition results in a treatment field that is not as extensive as other institutional practices, which may have contributed to the relatively low ilung doses obtained: other Post-M CTV definition methods may result in overall higher lung doses.

We, therefore, recommend that DIBH RT for patients who are treated Post-M and/or require SCV nodal coverage - particularly those with additional concomitant cardiopulmonary risk factors, including receipt of systemic therapies with known cardiac or pulmonary side-effects - should involve integrated planning and prescription processes, with careful individualized risk/benefit consideration of dose regimen and OAR. To determine optimal left-breast DIBH treatment with simultaneous nodal irradiation, further studies are required to investigate variations to target-volume definition, radiation schedules, and planning and delivery methods to minimize cardiac and pulmonary radiation, where planning methods that account for lung mass may benefit in the assessment of lung sparing..$^{14,21}$ 


\section{Conclusion}

The DIBH delivery technique is recommended as a feasible, effective protocol for optimal heart sparing in suitable patients. Selecting patients for DIBH at planning was effective, but the screening method showed insufficiencies. As a result, our screening protocol has evolved and patients are now selected for DIBH based on ability to perform adequate DIBH sufficient to undergo DIBH CT simulation, enabling more patients to receive subsequent DIBH treatment. Additional efforts to reduce lung sparing in Post-M/ SCV node patients are warranted that may improve overall outcomes following left-breast DIBH RT.

\section{Acknowledgments}

This study was undertaken as part of research and development and ongoing treatment assessment at The Harley Street Clinic within the auspices of HCA International - no specific funding source was used. The support of radiographers, medical physicists, dosimetrists, and radiation oncologists at The Harley Street Clinic Radiotherapy Department is gratefully acknowledged - particularly Andrew Edwards and Stephanie Houghton - for their contributions to the ongoing development of left-breast RT and the DIBH technique, successful treatment delivery, and helpful discussions.

\section{Disclosure}

The authors report no conflicts of interest in this work.

\section{References}

1. Darby SC, Ewertz M, McGale P, et al. Risk of ischemic heart disease in women after radiotherapy for breast cancer. $N \mathrm{Engl} \mathrm{J} \mathrm{Med}$. 2013;368(11):987-998.

2. Bhadra K, Patra NB, Manna A, Kabasi A, Pal J, Sarkar SK. Abnormalities by pulmonary regions studied with computer tomography and clinical correlation following local-regional radiotherapy for breast cancer. South Asian J Cancer. 2013;2(1):21-25.

3. Gokula K, Earnest A, Wong LC. Meta-analysis of incidence of early lung toxicity in 3-dimensional conformal irradiation of breast carcinomas. Radiat Oncol. 2013;8:268.

4. Henson KE, McGale P, Taylor C, Darby SC. Radiation-related mortality from heart disease and lung cancer more than 20 years after radiotherapy for breast cancer. Br J Cancer. 2013;108(1):179-182.

5. Krengli M, Sacco M, Loi G, et al. Pulmonary changes after radiotherapy for conservative treatment of breast cancer: a prospective study. Int J Radiat Oncol Biol Phys. 2008;70(5):1460-1467.

6. Latty D, Stuart KE, Wang W, Ahern V. Review of deep inspiration breath-hold techniques for the treatment of breast cancer. JMed Radiat Sci. 2015;62(1):74-81.

7. Smyth LM, Knight KA, Aarons YK, Wasiak J. The cardiac dose-sparing benefits of deep inspiration breath-hold in left breast irradiation: a systematic review. J Med Radiat Sci. 2015;62(1):66-73.

8. Mast ME, van Kempen-Harteveld L, Heijenbrok MW, et al. Left-sided breast cancer radiotherapy with and without breath-hold: does IMRT reduce the cardiac dose even further? Radiother Oncol. 2013;108(2):248-253.
9. Swanson T, Grills IS, Ye H, et al. Six-year experience routinely using moderate deep inspiration breath-hold for the reduction of cardiac dose in left-sided breast irradiation for patients with early-stage or locally advanced breast cancer. Am J Clin Oncol. 2013;36(1):24-30.

10. Vikström J, Hjelstuen MH, Mjaaland I, Dybvik KI. Cardiac and pulmonary dose reduction for tangentially irradiated breast cancer, utilizing deep inspiration breath-hold with audio-visual guidance, without compromising target coverage. Acta Oncol. 2011;50(1):42-50.

11. Osman SO, Hol S, Poortmans PM, Essers M. Volumetric modulated arc therapy and breath-hold in image-guided locoregional left-sided breast irradiation. Radiother Oncol. 2014;112(1): 17-22.

12. Remouchamps VM, Letts N, Vicini FA, et al. Initial clinical experience with moderate deep-inspiration breath hold using an active breathing control device in the treatment of patients with left-sided breast cancer using external beam radiation therapy. Int J Radiat Oncol Biol Phys. 2003;56(3):704-715.

13. Remouchamps VM, Vicini FA, Sharpe MB, Kestin LL, Martinez AA, Wong JW. Significant reductions in heart and lung doses using deep inspiration breath hold with active breathing control and intensity-modulated radiation therapy for patients treated with locoregional breast irradiation. Int J Radiat Oncol Biol Phys. 2003;55(2): 392-406.

14. Zurl B, Stranzl H, Winkler P, Kapp KS. Quantitative assessment of irradiated lung volume and lung mass in breast cancer patients treated with tangential fields in combination with deep inspiration breath hold (DIBH). Strahlenther Onkol. 2010;186(3):157-162.

15. Korreman SS, Pedersen AN, Aarup LR, Nøttrup TJ, Specht L, Nyström H. Reduction of cardiac and pulmonary complication probabilities after breathing adapted radiotherapy for breast cancer. Int J Radiat Oncol Biol Phys. 2006;65(5):1375-1380.

16. Krauss DJ, Kestin LL, Raff G, et al. MRI-based volumetric assessment of cardiac anatomy and dose reduction via active breathing control during irradiation for left-sided breast cancer. Int Jo Radiat Oncol Biol Phys. 2005;61(4):1243-1250.

17. Bruzzaniti V, Abate A, Pinnarò $\mathrm{P}$, et al. Dosimetric and clinical advantages of deep inspiration breath-hold (DIBH) during radiotherapy of breast cancer. J Exp Clin Cancer Res. 2013;32:88.

18. Nissen HD, Appelt AL. Improved heart, lung and target dose with deep inspiration breath hold in a large clinical series of breast cancer patients. Radiother Oncol. 2013;106(1):28-32.

19. Landberg T, Chavaudra J, Dobbs J, et al. ICRU Report 62: Prescribing and Reporting Photon Beam Therapy (Supplement to ICRU Report 50). J ICRU. 1999;os32(1):1-52.

20. Appelt AL, Vogelius IR, Bentzen SM. Modern hypofractionation schedules for tangential whole breast irradiation decrease the fraction size-corrected dose to the heart. Clin Oncol ( $R$ Coll Radiol). 2013;25(3):147-152.

21. Badiyan SN, Shah C, Arthur D, et al. Hypofractionated regional nodal irradiation for breast cancer: examining the data and potential for future studies. Radiother Oncol. 2014;110(1):39-44.

22. Matzinger O, Heimsoth I, Poortmans P, et al; EORTC Radiation Oncology \& Breast Cancer Groups. Toxicity at three years with and without irradiation of the internal mammary and medial supraclavicular lymph node chain in stage I to III breast cancer (EORTC trial 22922/10925). Acta Oncol. 2010;49(1):24-34.

23. Yeung R, Conroy L, Long K, et al. Cardiac dose reduction with deep inspiration breath hold for left-sided breast cancer radiotherapy patients with and without regional nodal irradiation. Radiat Oncol. 2015;10:200.

24. Rice L, Harris S, Green MML, Price PM. Deep inspiration breath-hold (DIBH) technique applied in right breast radiotherapy to minimize liver radiation. BJR Case Reports. 2015;2:20150038.

25. Hurt CN, Nixon LS, Griffiths GO, et al. SCOPE1: a randomised phase II/III multicentre clinical trial of definitive chemoradiation, with or without cetuximab, in carcinoma of the oesophagus. BMC Cancer. $2011 ; 11: 466$. 
26. Alderliesten T, Sonke JJ, Betgen A, Honnef J, van Vliet-Vroegindeweij $\mathrm{C}$, Remeijer P. Accuracy evaluation of a 3-dimensional surface imaging system for guidance in deep-inspiration breath-hold radiation therapy. Int J Radiat Oncol Biol Phys. 2013;85(2):536-542.

27. Cerviño LI, Gupta S, Rose MA, Yashar C, Jiang SB. Using surface imaging and visual coaching to improve the reproducibility and stability of deep-inspiration breath hold for left-breast-cancer radiotherapy. Phys Med Biol. 2009;54(22):6853-6865.

28. Hjelstuen MH, Mjaaland I, Vikström J, Dybvik KI. Radiation during deep inspiration allows loco-regional treatment of left breast and axillary-, supraclavicular- and internal mammary lymph nodes without compromising target coverage or dose restrictions to organs at risk. Acta Oncol. 2012;51(3):333-344.

29. Malin M, Kang H, Tatebe K, Hasan Y, Chmura S, Al-Hallaq H. SU-FJ-123: CT-based determination of DIBH variability and its dosimetric impact on post-mastectomy plus regional nodal radiation therapy. Med Phys. 2016;43(6):3435.

30. Wang W, Purdie TG, Rahman M, Marshall A, Liu FF, Fyles A. Rapid automated treatment planning process to select breast cancer patients for active breathing control to achieve cardiac dose reduction. Int $J$ Radiat Oncol Biol Phys. 2012;82(1):386-393.
31. Rochet N, Drake JI, Harrington K, et al. Deep inspiration breath-hold technique in left-sided breast cancer radiation therapy: evaluating cardiac contact distance as a predictor of cardiac exposure for patient selection. Pract Radiat Oncol. 2015;5(3):e127-e134.

32. Borst GR, Sonke JJ, den Hollander S, et al. Clinical results of imageguided deep inspiration breath hold breast irradiation. Int J Radiat Oncol Biol Phys. 2010;78(5):1345-1351.

33. Bartlett FR, Colgan RM, Carr K, et al. The UK HeartSpare Study: randomised evaluation of voluntary deep-inspiratory breath-hold in women undergoing breast radiotherapy. Radiother Oncol. 2013;108(2): 242-247.

34. Betgen A, Alderliesten T, Sonke JJ, van Vliet-Vroegindeweij C, Bartelink $\mathrm{H}$, Remeijer P. Assessment of set-up variability during deep inspiration breath hold radiotherapy for breast cancer patients by 3D-surface imaging. Radiother Oncol. 2013;106(2):225-230.

35. Haviland JS, Owen JR, Dewar JA, et al; START Trialists' Group. The UK Standardisation of Breast Radiotherapy (START) trials of radiotherapy hypofractionation for treatment of early breast cancer: 10-year follow-up results of two randomised controlled trials. Lancet Oncol. 2013;14(11):1086-1094.
Breast Cancer - Targets and Therapy

\section{Publish your work in this journal}

Breast Cancer - Targets and Therapy is an international, peerreviewed open access journal focusing on breast cancer research, identification of therapeutic targets and the optimal use of preventative and integrated treatment interventions to achieve improved outcomes, enhanced survival and quality of life for the cancer patient.

\section{Dovepress}

The manuscript management system is completely online and includes a very quick and fair peer-review system, which is all easy to use. Visit http://www.dovepress.com/testimonials.php to read real quotes from published authors. 